\title{
TRA LE RIGHE DEGLI ATTI DELLE CORTES DI MONZÓN DEL 1362-63*
}

\author{
Antonio Marongiu
}

«Cortes di Monzón») è un'espressione alquanto ellittica, la quale significa (o lo significherà per il periodo successivo a quello di cui si fa discorso) Cortes generali dei regni della Corona d'Aragona, in altre parole, come diceva di esse il 10 ottobre (a Nativitate Domini) il re Pietro IV, Corti (o Curiae) dei regni e territori cismarini'della Corona (ad esclusione, come non si mancava di affermare, della Sardegna e della Corsica) e del regno insulare di Maiorca. Il perché della sede (qualche volta contesa alla stessa Monzòn dalla non lontana Fraga) stava nella posizione geografica di confine tra l'Aragona e la Catalogna. Tali Corti erano dunque veramente generali e quadripartite, di Aragonesi, Catalani, Valenzani e Maiorchini. Ciascuno dei regni suddetti (quello di Catalogna, «principato», come gli altri) vi partecipava con

* Queste brevi pagine rappresentano un soltanto tenue omaggio ad un valoroso, eminente, Collega, quale storico delle istituzioni, ad illustre archivista, fino ad ieri direttore del prestigioso Archivio Generale della Corona d'Aragona, in Barcellona. Proprio da tale archivio e nella sua Colección de documentos inéditos (Segunda época) è venuto alla luce, come vol. I, a cura di J.M. PONS GURI, il volume, sul quale stiamo per intrattenerci. Actas de las Cortes Generales de la Corona de Aragón de 1362-63, Madrid, 1982, con Presentación dello stesso prof. F. UdINA MARTORELl e Introducción de PONS GURT. Nelle citazioni, ricorderemo il volume como Actas. 
le sue Cortes particolari, organizzate, come d'uso, su tre stamenti o bracci ciaseuna, quattro quelle aragonesi, per la separazione tra alta e bassa nobiltà. La già ricordata lettera circolare di convocazione' fu poi ripetuta, per la dichiarata impossibilità del Sovrano di essere a Monzón, per presiederle, il 4 novembre (dello stesso calendarjo). Rendeva informati i molti destinatari di ciascun paese e stamento che egli intendeva in dette curie, trattare con i suoi fedeli sudditi della guera già in atto con l'omonimo re Pietro di Castiglia, il quale, in violazione di ogni precedente accordo, aveva invaso l'Aragona, e dei sussidi necessari per la difesa della republica e la liberazione dei territori occupati. Intendeva, e lo precisava, trattare soltanto di ciò e non de aliis e chiedeva ed ammoniva (requirimus et monemus) d'intervenire alla riunione nel luogo, suddetto, e nella data indicata, infallibilmente (infallibiliter). Parimenti, impossibilitato egli medesimo a rispettare la data fissata, provvedeva poi, il 17 ottobre a fissare nuova data di comparizione, per il 10 novembre $^{2}$ diramando le opportune comunicazioni, regno per regno e braccio per braccio, a tutti i membri di ciascuno di essi, con nuovo comando e, ancora, l'avvertenza di comparire infallibiliter ${ }^{3}$.

La ragione, e il fine, della riunione si riduceva, come si era accennato, ad una sola, quella di procurare i mezzi per la prosecuzione della guerra contro gli aggressori castigliani: le Cortes dovevano trattare di questo, soltanto di questo - il Sovrano lo diceva e lo ripeteva- e non di altro ${ }^{4}$. Forse faceva bene a precisarlo, tenuto conto che, di solito, le Cortes dei paesi della Corona solevano, accanto ai problemi politici e finanziari, trattare anche ed ampiamente dei problemi della giustizia, dei greuges o gravamina, la cui trattazione o la risoluzione dei quali portava via assai spesso molto tempo. Le Curie o Cortes - sulle orme di antichi scrittori ed in particolare di $\mathrm{T}$. Mieres lo ha ricordato di recente il prof. J. Lalinde Abadía ${ }^{5}$ avevano per funzione spe-

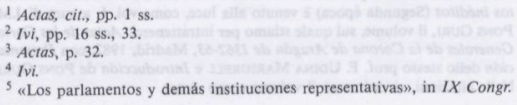


cifica di occuparsi, in generale, ed operare pro bono communi e per la utilitate totius reipublicae (a differenza dei «parlamentin chiamati a trattare di interessi specifici del Sovrano o del Paese). Abbiamo, dunque (ossia queste Cortes di Monzón avevano) una rigorosa ed imperiosa specificità di fini e di funzione: domandandocene il, pur ovvio, motivo dobbiamo opinare che il Sovrano volesse - infallibiliter, anche lui-condurre le cose con la maggiore possibile sollecitudine; tacesse, quindi, sul, pure consueto, giudizio sui gravami (o greuges) che fra bando a presentarli, nomine dei giudici e discussioni relative avrebbe (come, appunto, era uso nelle Cortes dei singoli regni, o peggio) provocato grave ritardo alla decisione ${ }^{6}$.

Che re Pietro urgesse per il pronto accoglimento della sua domanda lo dimostra tutto l'insieme degli Atti cui stiamo per accennare, ma forse, anche la circostanza, singolare, della identità della formula, egualmente imperativa (requirimus et monemus) rivolta tanto all'alto clero ed ai signori feudali quanto ai semplici cavalieri e alle "università», cioè città e ville reali, senza la consueta deferenza verso, appunto, gli ecclesiastici, tanto sensibili e desiderosi di preghiere anziché di comandi'.

Stor. Cor. Arag. La Corona d'A ragona e il Mediterraneo, Aspetti e problemi comuni, Napoli 1973, Napoli 1978, p. 148, con riferimento anche a T. MiERES, Apparatus super constitutionibus curiarum generalium Cathaloniae, p. 2, Barcelonae 1621 .

${ }^{6}$ Storici frettolosi o distratti sottovalutano, oppure ignorano del tutto, la funzione precipua dei parlamenti medievali (inglese e altri) di chiedere e fare giustizia (in buona parte politico-amministrativa). Su tale funzione insisto nel mio Saggio Introduttivo, in corso di stampa, alla edizione degli Atti dei parlamenti sardi.

${ }^{7}$ Cfr. A. Marichalar \& C. Manrique, Historia de la legislación y recitaciones del derecho civil de España, vol. 9, Madrid 1861-72, vol. 7, pp. 200 ss. Nel parlamento siciliano (anche la Sicilia appartenne a lungo alla Corona d'Aragona) l'invito era formulato come esortazione se rivolto ai prelati ed ecclesiastici, come «incarico" se rivolto ai membri del braccio «militaren e come comando se rivolto alle città e ville «reali». Anche in Sardegna, p. es., la convocatoria per il parlamento del $1553-54$ al braccio ecclesiastico diceva vos pregam, exortam y citam, e quelle ai Militari e alle città e ville vos citam y manam. Talvolta, però, la formula era eguale per tutti e tre e ciò provocava proteste. 
Unitaria, identica, era la formulazione dell'invito fatto ai capitoli cattedrali (per i quali si parlava di yconomos) alle città e ville, per le quali si parlava di sindicos e procuratores ed ai maiorchini tutti (che dovevano, come abbiamo già accennato, affrontare il viaggio in mare) a scegliere e mandare persone di loro fiducia, timorose di Dio, amanti del Sovrano e del Paese e munite di pieno potere a trattare, fare e firmare ciò che stava o doveva esser fatto e stabilito sulla questione della guerra di liberazione dei territori occupati da parte dei Castigliani. Notevole, del tutto eccezionale è la circostanza, rilevata dall'editor degli Atti J.M. Pons Guri ${ }^{8}$, dell'assenza di contestazioni o rilievi sulla sufficienza o meno dei mandati realmente conferiti a tali economi, sindaci o procuratori: forse, essi furono, davvero, pieni e bastanti. Eे pure singolare il fatto che, pur accennandosi a qualche ritardato arrivo non si parlasse di una eventuale dichiarazione di contumacia degli assenti, cosa, peraltro, consueta nelle riunioni delle Cortes iberiche ${ }^{9}$. Si preferi, probabilmente, appunto non indugiare su tali circostanze: del resto anche la verifica dei mandati e le «abilitazioni» dei convenuti avrebbero potuto anch'esse far perdere tempo. Il Re - esperto di negozi

Chiaramente, fin quasi ai nostri giorni, la convocazione delle sessioni parlamentari era, quasi da per tutto, prerogativa sovrana.

${ }^{8}$ Actas, cit., p. XVI: «llega al extremo de prescindir de los instrumentos de mandato de quienes se personan mediante apoderado, del bastanteo de los poderes, estimación de excusas y datos concernientes a los convocados a cortes, sobre cuyos extremos son tan profusos otros procesosm.

${ }^{9}$ Normalmente, tre successive proroghe, secondo una tradizione vagamente romanistica e canonistica che faceva delle convocatorie alle riunioni parlamentari degli ordini a comparire davanti al giudice. Questo nesso tra le convocazioni parlamentari e le citazioni giudiziali è stato, com'è noto, messo in evidenza da GAINES POST, nello scritto Roman law and early representation in Spain and Italy, in «Speculam", XVIII (1943), ora nel volume Studies in medieval legal thought, Public Law and the State, 1100-1322, Princeton 1964, pp. 61 ss.

La formula unitaria requirimus et monemus fu impiegata dallo stesso Pietro IV d'Aragona anche nel convocare le Cortes aragonesi, il 22 gennaio 1375: cfr. L. Ledesma RuBio, Actas de proceso de Cortes de Tamarite de 1375, Zaragoza 1979, pp. 12 ss. 
parlamentari- sapeva il fatto suo ed agiva in prima persona; era lui che convocava e rinviava le assemblee, che le presiedeva, che agiva e trattava in prima persona, dal principio alla fine. I sudditi sembravano, del resto, pieni anch'essi di zelo e di buona volontà di collaborazione ${ }^{10}$.

Riunita, finalmente (non era poi passato gran tempo neppure dalla prima convocazione), l'assemblea, senza perdere tempo nelle, pur consuete, discussioni sui posti da occupare (non sarebbe forse assurdo pensare che rimanessero tutti in piedi!), il 23 novembre" , il Re rivolse ai presenti la sua breve allocuzione, o proposicio, con linguaggio attinto dalle fonti bibliche, in una sala del castello di Monzòn, di cui erano ospiti. Anziché - come sarebbe stato negli usi-il più elevato in grado degli ecclesiastici presenti, gli rispose, a nome di tutti illi de curia suo fratello, l'infante Ferdinando, marchese di Tortosa, con la consueta assicurazione che essi avrebbero provveduto e deliberato quanto sarebbe loro apparso piú conveniente nell'interesse del Re e del Regno. Qualche giorno dopo, entravano in scena i «trattatori» (non risulta come eletti né in quale numero e se tutti di nomina regia o pure mista) e diventava, in certo senso, di pubblico dominio che la decisione da adottare concerneva il contenuto e le modalità degli accordi necessari a chiamare in Aragona il condottiero conte di Trastamara e il Maresciallo di Francia, con i loro armigeri, per opporli agli invasori castigliani. Tutto si riduceva, pertanto, al problema di approntare i mezzi necessari per assoldarli ed anche consistenti contingenti di truppe a cavallo ed a piedi per fiancheggiarli, e, naturalmente, provvedere a ripartire tali oneri fra i gruppi presenti o rappresentati nelle Cortes: per il regno di Maiorca bisognava anche provvedere alla ripartizione tra l'isola maggiore e quelle minori.

Tutto procedette nel migliore dei modi. Il $1^{\circ}$ dicembre il $\mathrm{Re}$ s'incontrava con i trattatori per informarli dello stato delle trattative con il Trastamara e, non molto dopo, due ecclesiastici esprimevano il consenso di massima della «curia». Per far le cose

10 Actas, p. 54.

11 Ivi., p. 55. 
in regola bisognò però provvedere, il 4 febbraio $1363^{12}$, ad un allargamento e ad una migliore distribuzione dei trattatori, per i vari regni e stamenti. Ben 33 parlamentari, in rappresentanza, appunto, dei vari regni e gruppi, ricevettero pertanto l'incarico di provvedere super modo solvendi et habendi quantitatem subsidii faciendi ad prosecutionem guerre, et defensionem reipublice regnorum et terrarum domini regis: notevoli, in ciò, due cose, entrambe fuori dall'ordinario, il gran numero di questi trattatori ed il fatto che venissero nominati non pariteticamente, ma soltanto da parte dei bracci. Tali trattatori giurarono ${ }^{13}$, com'era ovvio, di bene a fedelmente adempiere al loro ufficio, sul Vangelo: non giurarono (com'era loro regola) quelli ecclesiastici. Dato l'impegno e la buona volontà di sbrigar tutto bene e sollecitamente i lavori procedettero senza ostacoli. Il 6 febbraio 1363 , nessuna obiezione essendo stata sollevata contro la richiesta regia, i trattatori avevano già deciso e l'ammontare dell'aiuto o sussidio (gli Atti parlano anche di «dono grazioso», cioè di prestazione del tutto volontaria, non dovuta) e il modo della riscossione, da farsi per fogatge o fuocatico, cioè «fuoco» per «fuoco», ed anche l'invito al Sovrano a provvedere alla ripartizione fra i vari regni del suo ammontare di 250.000 libre, o lire. Presenti, allora, omnes illi de curia, il Re leggeva allora la sua distributio $^{14}$.

Tutto l'insieme fu approvato per acclamazione, senza alcuna formalità di voto, da tutti. Il Re aveva domandato, domandava, se la Cort fosse d'accordo sull'aiuto e sulla distributio da lui operata e tutti i presenti, interrogati, appunto, si omnia predicta placebant eis, rispondevano (tutti o almeno plures) «tumultuosamenten e ripetutamente il loro oc, oc esprimente consenso. Sabato 11 febbraio, in una nuova assemblea, lo stesso Sovrano lamentava dei contrasti tra le varie parti, ciascuna delle quali avrebbe preteso di riversare su altre parte dei loro oneri, mentre il pericolo era ancora grave ed urgente e il «dono» (lo do) non era sta-

12 Ivi., p. 58.

13 Ibidem, p. 60.

14 Ivi., p. 69. 
to ancora fatto (atorgat) ${ }^{15}$. Sedati, per l'autorevole mediazione regia, i residui dissensi o contrasts, domenica 5 marzo $^{16}$, lo stesso Sovrano chiedeva agli intervenuti una nuova conferma degli accordi pattuiti: nuovo voto per acclamazione e tutti o quasi tutti (quamplures) esprimevano ancora una volta, semel et simul, il loro benestare (Plau nos, Senyor!), mentre alcuni di loro, proferendo il tradizionale $o c$, $o c$ alzavano, tra le mani, copie degli accordi già sottoscritti: oblatio, da un lato, e conditiones, dall'altro, cioè i patti ai quali la stessa offerta veniva legata ${ }^{17}$, insomma la forma ac modus donorum, messe per scritto con atto pubblico. Mancava la parola «contratto», ma la sostanza era proprio questa.

In realtà, e ciò risultava ampiamente dai ${ }^{18}$ Capitula doni facti per Generale Catalonie regnique Maioricarum et insulas eidem regno adiacentes e dagli analoghi documenti degli altri paesi, il donum et subsidium concessi al Sovrano erano stati molto meno "graziosi») o gratuiti di quanto tuttavia si insistesse nel ripetere... Durante le precedenti settimane, i trattatori dei vari paesi avevano infatti preparato una lunga serie di richieste da sottoporre al Sovrano in cambio, appunto, del «dono»: si trattava perciò, hinc inde, di un do ut des, di un dare per avere ${ }^{19}$. Prezzo pagato (senza questa... brutta parola) a favore dei Catalani erano ben 62 capitoli, che il Re sanciva (reb agradablement) a loro richiesta, toccanti la politica economica, gli scambi di materie prime e di manufatti, il commercio, la finanza, la tutela della produzione agricola, l'osservanza, in genere, dell'ordinamento giuridico, dei «privilegi», del paese, cioè delle antiche Costituzioni: degna di nota anche la premessa di tali capitoli, che, cioè, il Re, non bastandogli, alla difesa del territorio, il suo patrimonio, aveva accondisceso ad accettare il dono, graziosamente (no per deute qu'en fossen tenguts e obligats), gli stessi Catalani ${ }^{20}$.

\footnotetext{
15 Ancora Actas, p. 63 s.

16 Ivi., p. 69.

17 Actas, pp. 69 ss.

18 Testo, ivi., pp. 70-104.

19 Ivi., p. 72.

20 Ancora Actas, pp. 104 ss.
} 
Con essi e verso tutti gli altri s'impegnava a non interferire minimamente con i suoi dipendenti sulla riscossione dello stesso dono, che doveva essere sospeso o smesso in caso di tregua o di pace. In parte simili a quelli catalani, in parte diversi, meno arnpi quelli relativi alla politica mercantilistica di protezionismo economico e più diffusi su altri punti relativi, in specie, al servizio militare, ma pure ben accolti dal Sovrano, furono, e sono agli Atti, i 39 capitoli dei tre bracci privilegiati (prelati ed ecclesiastici, richos homens e cavallers) aragonesi, puntuali tutti nel precisare e circoscrivere gli obblighi da essi assunti ${ }^{21}$. Il «dono» del regno di Valenza era... alleggerito da ben 56 capitoli del più vario contenuto $^{22}$ e dalla richiesta di conferma (come i Catalani facevano delle proprie Costituzioni) dei suoi antichi usi e furs ${ }^{23}$.

Come a coronamento di tutto ciò, 1'8 marzo 1363, lo stesso re Pietro, nel corso di una nuova assemblea generale, di omnes de curia, rinnovava a tutti i suoi ringraziamenti per il dono che essi avevano fatto a se stesso ed allo Stato - sibi et reipublice-, che gli avrebbe consentito, diceva, di riunire truppe sufficienti a sconfiggere e respingere il nemico ${ }^{24}$. Giurava ${ }^{25}$ di osservare i capitoli stabiliti ed accettava di buon grado la minaccia di scomunica comminatagli, per il caso di trasgressione di tali accordi, dal primate arcivescovo di Tarragona. Dopo di ciò, licenziava la curia $^{26}$, ma, con «atto di benignità», consentiva che nei prossimi giorni, fino alla domenica 11 marzo compresa, chiunque potesse esporre i suoi eventuali gravami (fino ad allora non se ne era, sembra, parlato) al Vicecancelliere, venerabile Francesco Roma, dottore in diritto, che ne avrebbe fatto giustizia favorabiliter et benigne, con decisioni valide come se fossero state prima dela chiusura delle Cortes $^{27}$. Fatto conclusivo, tutta una lunga serie di sottoscrizioni degli intervenuti. Agli Atti, o reso-

\footnotetext{
${ }^{21}$ Ivi., p. 115 ss.

22 Ibidem, pp. 127 ss.

23 Actas, pp. 171 ss.: Furs in loch de Constitutions de Catalunya.

24 Actas, p. 196.

25 Ivi., p. 197.

26 Quibus sic peractis, licentiavit Curiam.

27 Ancora Actas, Ic. cit.
} 
conti fin qui riferiti seguivano, infine, ordinazioni e capitoli recanti particolarità operative delle riscossioni da fare ed altre analoghe scritture, e norme particolari dettate dal Re sulle attività giudiziarie nella città di Barcellona ${ }^{2 \hbar}$ e sulle concessioni di generositas nel regno di Valenza ${ }^{29}$.

I testi raccolti fra le Actas sono variamente interessanti (redatti, come sono, in latino e nei linguaggi dei differenti paesi) anche sotto il profilo linguistico, oltre che per i ripetuti $o c$, $o c$ di consenso, per l'apparizione, ripetuta, della parola condicio come sinonima di stament $^{30} \mathrm{e}$ la qualifica dell'appartenenza allo stamento militare o di quest'ultimo, come un genus ${ }^{31}$. Non sono però più importanti di altre caratteristiche già rilevate, per essempio delle diciture degli inviti, l'assenza di minaccia di dichiarazione di contumacia degli assenti e della verifica dei poteri e mandati e di un adeguato giudizio sui greuges. Visti e seguiti giorno per giorno, i rapporti tra il Sovrano e i regni e bracci convocati sembrano essere stati perfetti e, per cosi dire, ideali, ottimi, come manifestazioni, addirittura, di un vero e proprio regime parlamentare. Le Cortes facevano ciò che proponeva il Sovrano e questi sanzionava e giurava le richieste di essi. Una cosa, però, non può non essere taciuta ed è la seguente. Secondo il diritto pubblico generale, negli Stati medievali a struttura feudale, i vassalli non dovevano al Signore se non ciò cui erano obbligati dai rispettivi patti e se il sovrano voleva qualche cosa d'altro o di più del pattuito, egli doveva chiedere il permesso degli stessi vassalli. Però questa regola subiva eccezione nei tradizionali «tre casi» 0 "quattro casi», d'invasione o grave minaccia d'invasione del territorio, di prigionia del sovrano, e di nuova milizia sua o matrimonio di sua figlia: casi, nei quali, appunto il Signore poteva imporre ed esigere dai vassalli veri e propri tributi. Forse la tes-

28 Actas, pp. 210 ss.

29 Ivi., pp. 217 ss.

${ }^{30}$ Di condicio o stament si parla a pp. $72,74,75$, ma $c f r$. anche il mio recente studio Estament, bras o condicio nelle Corts catalane, estr. «Bolet. Faculd. Direito de Coimbra-Est. homen. M.P. Merêa e G. Braga da Cruz», Coimbra 1983.

31 Actas, pp. 49-50. 
timonianza più precisa di tale norma è la Costituzione per il regno di Sicilia dell'alto Signore di questo Onorio papa IV, nella Edictalis provisio, del 1285, contenuta appunto nei Capitula papae Honorii $^{32}$. A mente di tali principi, Pietro IV d'Aragona avrebbe potuto procedere a tassare, per la liberazione del territorio invaso, i sudditi, senza bisogno di chiedere un'apposita concessione di essi a Cortes.

Perché non l'avrà fatto forse il suo Stato mancava degli strumenti necessari per una simile imposizione generale o forse egli temeva che Catalani, Aragonesi e Maiorchini gli rispondessero che, in fin dei conti, era invaso il solo territorio aragonese e che quindi egli dovesse rivolgersi unicamente ai sudditi aragonesi?

Egli ritenne, come si è visto, miglior partito il ricorso ad una convocazione generale, sollecita, senza greuges. I fatti gli dettero ragione.

${ }^{32}$ Cfr. A. MARongiU, La Charte des libertés siciliennes de 1285, "Album E. Màlysuz = Et; Commiss. intern. Hist. Ass. 2 t., LVI, Bruxelles 1976 , pp. 171 ss. 